\title{
Patients with systemic sclerosis-associated pulmonary arterial hypertension express a genomic signature distinct from patients with interstitial lung disease
}

Journal of Scleroderma and Related Disorders 2018, Vol. 3(3) 242-248 (C) The Author(s) 2018 Article reuse guidelines: sagepub.com/journals-permissions DOI: $10.1177 / 2397198318764780$ journals.sagepub.com/home/jso (S)SAGE

\author{
Matthew Moll', Romy B Christmann2, Yuqing Zhang, \\ Michael L Whitfield ${ }^{5}$, Yu Mei Wang², Lisa Rice'2, \\ Eric Stratton ${ }^{2}$, Robert Lafyatis ${ }^{2,6}$ and Harrison W Farber ${ }^{7}$
}

\begin{abstract}
Objective: Pulmonary arterial hypertension and interstitial lung disease are major causes of mortality in systemic sclerosis. We used a previously identified microarray biomarker to determine whether systemic sclerosis-pulmonary arterial hypertension and systemic sclerosis-interstitial lung disease patients demonstrate distinct gene expression profiles. Methods: Peripheral blood mononuclear cells were collected from healthy controls $(n=10)$, systemic sclerosis patients without pulmonary hypertension (systemic sclerosis-no pulmonary arterial hypertension, $n=39$ ), and systemic sclerosispulmonary arterial hypertension patients $(n=21$; mean pulmonary arterial pressure $\geq 25$, pulmonary capillary wedge pressure $\leq 15$, and pulmonary vascular resistance $\geq 3$ Wood units) diagnosed by right heart catheterization. Systemic sclerosis-interstitial lung disease patients were defined as those with evidence of fibrosis on chest computed tomography and significant restriction (forced vital capacity $<70 \%$ predicted, $n=I I$ ). Systemic sclerosis-pulmonary arterial hypertension biomarker included 69 genes selected by unbiased statistical screening of three publicly available microarray studies. RNA levels were measured by NanoString Technologies. Gene expression levels that were significantly correlated with pulmonary arterial hypertension (multiple statistical measures) were chosen as inputs into a forward selection logistic regression model.

Results: When interstitial lung disease patients were included $(n=64)$, four genes (SI00P, CD8BI, CCL2, and TIMPI) and male sex predicted pulmonary arterial hypertension with a high level of accuracy (area under the curve $=0.83$ ). Without interstitial lung disease patients $(n=53)$, two genes (THBSI and CD8BI) and male sex predicted pulmonary arterial hypertension with a high level of accuracy (area under the curve $=0.80$ ). When examining systemic sclerosis patients with borderline elevated pulmonary pressures (mean pulmonary arterial pressure $=2 \mathrm{I}-24 \mathrm{mmHg}$ ), gene expression changes closely resembled the systemic sclerosis-pulmonary arterial hypertension group, except for THBSI. Conclusion: Systemic sclerosis-pulmonary arterial hypertension and systemic sclerosis-interstitial lung disease have similar but distinct gene expression profiles. Many gene expression changes occur early in the disease course, potentially allowing early detection. THBSI appears to be an important mediator in the development of pulmonary arterial hypertension-predominant phenotype. Further prospective investigation is warranted.
\end{abstract}

\footnotetext{
'Division of Pulmonary and Critical Care Medicine, Brigham and Women's Hospital, Boston, MA, USA

2Division of Rheumatology, Department of Medicine, Boston University School of Medicine, Boston, MA, USA

${ }^{3}$ Division of Rheumatology, Allergy and Immunology, Massachusetts General Hospital and Harvard Medical School, Boston, MA, USA

${ }^{4}$ Clinical Epidemiology Research and Training Unit, Boston University School of Medicine, Boston, MA, USA

${ }^{5}$ Department of Molecular and Systems Biology, Geisel School of Medicine at Dartmouth, Hanover, NH, USA

${ }^{6}$ Division of Rheumatology and Clinical Immunology, Department of Medicine, University of Pittsburgh Medical Center, Pittsburgh, PA, USA

${ }^{7}$ Pulmonary Center, Department of Medicine, Boston University School of Medicine, Boston, MA, USA
}

Corresponding author:

Matthew Moll, Division of Pulmonary and Critical Care Medicine, Brigham and Women's Hospital, Boston, MA 02I I5, USA.

Email: mmoll@partners.org 


\section{Keywords}

Systemic sclerosis, interstitial lung disease, pulmonary

arterial hypertension, biomarker, genomic, gene

expression

Date received: 3 November 2017; accepted: 2I February 2018

\section{Introduction}

Pulmonary arterial hypertension (PAH) and interstitial lung disease (ILD) are major causes of morbidity and mortality in systemic sclerosis (SSc); ${ }^{1}$ therefore, early detection of either PAH and/or ILD could allow intervention and alteration of disease course.

The World Health Organization (WHO) classifies pulmonary hypertension $(\mathrm{PH})$ into five groups on the basis of mechanism: ${ }^{2} \mathrm{WHO}$ group I is classified as $\mathrm{PAH}$, defined as a mean pulmonary arterial pressure $(\mathrm{mPAP}) \geq 25 \mathrm{mmHg}$ at rest, a mean pulmonary capillary wedge pressure or left ventricular end-diastolic pressure $\leq 15 \mathrm{mmHg}$, and a pulmonary vascular resistance (PVR) $\geq 3$ Wood units (WU). Group 1 includes idiopathic PAH (IPAH), heritable PAH, PAH associated with disease states such as collagen vascular disease, PAH associated with drugs and toxins, portal hypertension, congenital left-to-right intracardiac shunts, human immunodeficiency virus, and schistosomiasis. Other less common subgroups include persistent $\mathrm{PH}$ of the newborn (PPHN), pulmonary venoocclusive disease (PVOD), and so on. ${ }^{3}$ The histopathologic hallmark of PAH is increased pulmonary arterial intimal thickness, vascular smooth muscle cell proliferation, thrombosis, and plexiform lesions; these changes eventually lead to right ventricular remodeling, failure, and death. ${ }^{3}$

SSc-associated PAH (SSc-PAH) is classified as group I $\mathrm{PH}$, but $\mathrm{PH}$ can also occur in the context of interstitial fibrosis (WHO group III) or left heart failure (WHO group II) in SSc patients. One hypothesis is that SSc-PAH (WHO group I) is driven by inflammation and immune dysregulation (e.g. macrophage activation and TGF- $\beta^{4}$ ), while SSc-associated ILD and PH (SSc-ILD-PH) is driven by obliteration of pulmonary capillaries with resultant increase in PVR. Theoretically, SSc patients can have PAH alone, ILD alone, or both PAH and ILD; in reality, most SSc patients have some degree of fibrosis. However, it is unknown whether in SSc patients with ILD causing significant restriction (forced vital capacity (FVC) $<70 \%$ ), development of PAH occurs by differing mechanisms than in patients with more mild interstitial disease. This is important to clarify because SSc patients could have different molecular phenotypes and, thus, may require separate clinical classifications in trials, molecular diagnostics, and therapeutic targets.

Previously, we identified a microarray-derived biomarker gene-cluster from SSc-PAH patients that correlated with mortality. 4,5 We further developed this biomarker using a reliable and quantitative method of RNA expression analysis in order to have a limited but more specific number of genes to determine whether, at a molecular level, SSc patients with PAH were distinct from those with ILD and significant restriction.

\section{Methods}

The Boston University Medical Center Institutional Review Board reviewed and approved the conduct of this study. Informed consent was obtained from all patients and healthy subjects. Patients selected for this study included patients with SSc without PH (SSc-noPAH), $n=39$ ), SSc with PAH (SSc-PAH, $n=25)$, and healthy controls $(n=10)$.

SSc disease duration was measured from the onset of the first non-Raynaud's phenomenon symptom. Patients with SSc were diagnosed with PAH by right heart catheterization (RHC): $\mathrm{mPAP} \geq 25 \mathrm{mmHg}$, pulmonary arterial wedge pressure (PAWP) $\leq 15$, and PVR $\geq 3 \mathrm{WU}$. All RHCs were performed according to standard protocol by a single individual (HWF). Pressure transducer was zeroed to the left atrium, and all measurements recorded at end-expiration and averaged over three respiratory cycles. Cardiac output was calculated by both thermal dilution and estimated Fick methods. Significant ILD was diagnosed by a predicted $\mathrm{FVC}<70 \%$ and review of chest computerized tomography to ensure there was significant evidence of fibrosis (reticulations, ground glass opacities, honeycombing, and septal thickening) and no alternative explanation for restriction (SSc-PAH $(n=3)$ and SSc-noPAH $(n=8)$ ).

Blood was collected from healthy controls and patients either on the day of the catheterization or within 3 months in cell preparation tubes (CPT) tubes designed for one-step cell separation (Becton Dickinson). Samples were immediately centrifuged and peripheral blood mononuclear cells (PBMCs) were isolated and total RNA was extracted using RNeasy ${ }^{\circledR}$ Mini Kits (Qiagen) according to the protocol of the manufacturer. RNA samples were stored at $-80 \mathrm{C}$.

RNA (100ng) from each sample was used for the mRNA analysis using NanoString technology. NanoString technology uses two hybridization probes of at least $50 \mathrm{bp}$, one of which has a "barcode" that is unique to the target mRNA, and the other allows immobilization of target transcripts. Excess probes are washed off and the number of unique hybridized barcodes is detected by nCounter technology without DNA amplification; the lack of amplification results in data with low background noise, and improves sensitivity and reproducibility. The PAH gene panel was built based on three publicly available microarray studies ${ }^{4,5}$ on PBMC from patients with SSc-PAH, SScnoPAH, and healthy controls.

Gene selection process: the PAH panel was built based on microarray analysis of PBMCs from SSc-PAH patients compared to SSc-noPAH and controls, previously published. ${ }^{4} \mathrm{~A}$ total of 69 genes were defined as SSc-PAH biomarker panel based on statistical power and biological rationality. 
Table I. Demographic information of patients, stratified by presence or absence of PAH.

\begin{tabular}{llll}
\hline & SSc-noPAH $(n=39)$ & SSc-PAH $(n=25)$ & $p$ value \\
\hline Age (mean) & 62.3 & 66.8 & 0.17 \\
Limited/diffuse & $38 / 01$ & $21 / 04$ & \\
Scl-70+ & 3 & 0 & \\
Anti-centromere+ & 1 & 3 & $<0.001$ \\
DLCO (mean, \%) & 65.1 & 45.2 & 0.06 \\
FVC (mean, \%) & 93.7 & 82.6 & \\
\hline
\end{tabular}

PAH: pulmonary arterial hypertension; SSc: systemic sclerosis; DLCO: diffusing capacity of the lungs for carbon monoxide; FVC: forced vital capacity.

Normalization was performed using housekeeping genes that were similar among all groups regardless of the presence of SSc disease and cardiopulmonary complications.

\section{Statistical analysis}

\section{nCounter expression analysis}

Expression analysis of the selected genes was investigated by nCounter expression analysis. The workflow is described at http://www.nanostring.com/elements/workflow. Background correction and normalization of data were performed using the nSolver Analysis Software 3.0 (NanoString Technologies).

\section{Correlation to PAH}

To determine which genes predict $\mathrm{PAH}$, gene expression levels were divided into quartiles and correlated to the percentage of PAH patients in each cohort; a linear relationship was determined by the $p$-value of $<0.05$ for the Pearson correlation coefficient.

\section{Correlation of genes with each other}

The set of genes that correlated with PAH presumably represent coordinated expression of multiple genes within a biologic pathway. Therefore, the genes that were significantly associated with PAH were correlated with each other, as measured by Pearson correlation coefficient $(r \geq 0.5)$. For each set of genes that were correlated, one to two genes were chosen to represent a group of correlated genes, based on a combination of the highest $r$-value, highest fold-change, highest c-statistic for predicting $\mathrm{PAH}$, and biologic plausibility.

\section{Logistic regression model}

The genes chosen by the above process were then used as inputs into a stepwise forward selection logistic regression model and the final model was chosen based on c-statistic. Sex was added as an input as male sex has been associated with PAH severity. ${ }^{6}$ Receiver operating characteristic (ROC) curves were used to compare the models for the cohorts with and without ILD patients.

\section{Analysis of borderline elevated PAP patients}

To understand whether gene expression changes identified by logistic regression analysis occur early in disease course, we plotted gene expression levels based on the following groups: healthy controls $(n=10), \mathrm{SSc}-$ noPAH low (mPAP $<20 \mathrm{mmHg}, n=28$ ), SSc-noPAH high (mPAP 21$24 \mathrm{mmHg}, n=7)$, and SSc-PAH ( $n=18)$. One-way analysis of variance (ANOVA) tests were used to determine statistical significance across groups.

\section{Results}

Patient characteristics are displayed in Table 1. Age was similar between those with and without PAH. There was significantly lower diffusing capacity of carbon monoxide (DLCO) and a trend toward restriction (reduced FVC) in the SSc-PAH group $(p=0.06)$. RHC was not performed in all patients without $\mathrm{PAH}$, so we cannot compare the data statistically; however, in the SSc-noPAH group with RHC data $(n=19)$, mPAP was $20.0 \pm 3.0 \mathrm{mmHg}$, mean cardiac index (Fick) was $3.16 \pm 0.57 \mathrm{~L} / \mathrm{min} / \mathrm{m} 2$, and mean PVR was $2.08 \pm 0.62 \mathrm{WU}$. In the SSc-PAH group, the mPAP was $37.4 \mathrm{mmHg} \pm 9.3 \mathrm{mmHg}$, mean cardiac index was $2.91 \pm 0.94 \mathrm{~L} / \mathrm{min} / \mathrm{m} 2$, and mean PVR was $7.04 \pm 4.38 \mathrm{WU}$.

Data were analyzed with and without SSc-ILD patients. Gene expression levels were correlated to percentage of PAH patients by Pearson correlation coefficient (Figure 1(a), representative example). When all SSc patients were included (SSc-noPAH without ILD $(n=35)$, SSc-noPAH with ILD $(n=8)$, SSc-PAH without ILD $(n=18)$, SScPAH-ILD $(n=3)), 14$ genes were significantly correlated with PAH (ADM, BCAT1, FBP1, MS4A4A, S100A10, S100P, THBS1, CD8B1, MSH2, CCL2, CEP1, JAK2, TIMP1, and PCGF5). These genes were then correlated to each other, and based on multiple statistical criteria and biologic plausibility (see section "Methods" for details), five genes (S100P, TIMP1, CCL2, THBS1, CD8B1) and sex were chosen as inputs into a stepwise forward selection logistic regression model; the final model included S100P, CD8B1, CCL2, TIMP1, and sex $(\mathrm{AUC}=0.83$, Figure 1(c)). S100P, CCL2, and TIMP1 were positively correlated with PAH. CD8B1 was negatively correlated with PAH. Male sex correlated with PAH. 


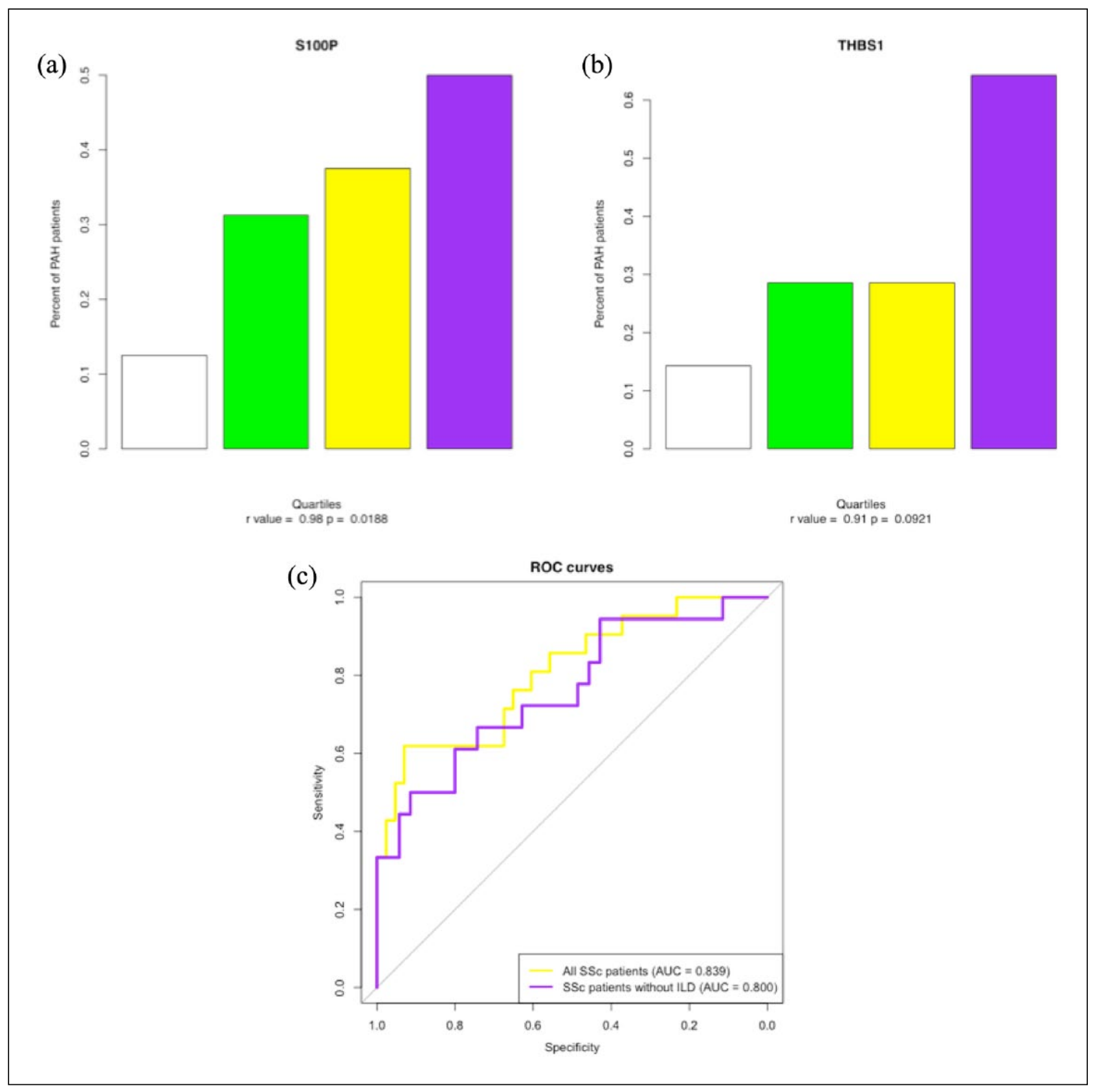

Figure I. (a) Representative example of gene expression levels correlating to PAH incidence (SI00P with all patients included). (b) Representative example of gene expression levels correlating to PAH incidence (THBSI from cohort excluding ILD patients with significant restriction). (c) ROC with all subjects and excluding subjects with ILD.

When patients with significant ILD were excluded, and only SSc-noPAH without ILD $(n=35)$ and SSc-PAH without ILD $(n=18)$ patients were compared, nine genes were strongly correlated to PAH (Figure 1(b), representative example), which included ADM, BCAT1, MS4A4A, S100A10, THBS1, S100P, CD8B1, MSH2, and TIMP1. These genes were then correlated to each other, and based on a variety of statistical criteria and biologic plausibility (see section "Methods" for details), three genes (S100P, THBS1, and CD8B1) and sex were chosen as inputs into a stepwise forward selection logistic regression model; the final model included THBS1, CD8B1, and sex (AUC $=0.80$, Figure 1(c)). THBS1 was positively correlated with $\mathrm{PAH}$ and $\mathrm{CD} 8 \mathrm{~B} 1$ was negatively correlated with PAH. Again, male sex was correlated with PAH.

Ideally, gene expression changes that predict the development of PAH would occur early in the disease course, allowing earlier detection; if gene expression in SScnoPAH high (mPAP 21-24 mmHg) and SSc-PAH patients change in parallel, such changes could represent early 


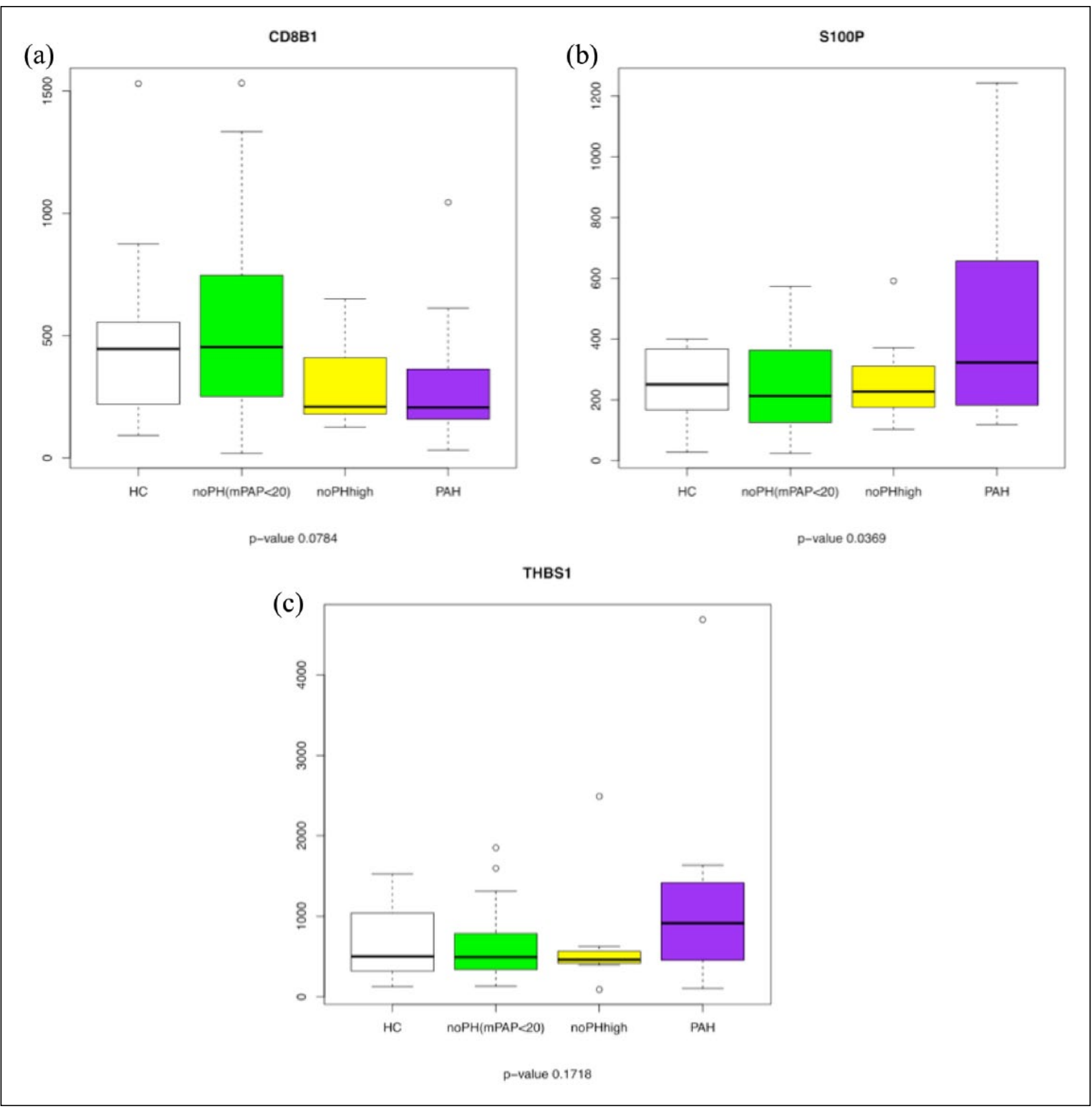

Figure 2. (a)-(c) Gene expression for three genes grouped by healthy controls $(n=10)$, SSc-noPAH low $(\mathrm{mPAP} \leq 20 \mathrm{mmHg}$, $n=28$ ), SSc-noPAH high (mPAP $2 \mathrm{I}-24 \mathrm{mmHg}, n=7)$, and SSc-PAH $(n=18)$. Analysis of variance (ANOVA) tests were used to determine statistical significance across groups.

CD8BI: cluster of differentiation 8-BI; SI00P: SI00 calcium-binding protein P; THBSI: thrombospondin I.

changes in PAH pathogenesis. In Figure 2(a)-(c), we show gene expression levels for S100P, THBS1, and CD8B1 for healthy controls $(n=10)$, SSc-noPAH low (mPAP $<20 \mathrm{mmHg}, \quad n=28)$, SSc-noPAH high (mPAP 21$24 \mathrm{mmHg}, n=7)$, and SSc-PAH $(n=18)$ patients. S100P and CD8B1 gene expression levels in the SSc-noPAH high group closely reflected changes in the SSc-PAH group; there was a statistically significant trend from healthy controls to SSc-PAH $(p<0.05)$. In contrast, THBS1 gene expression was not similar between the SSc-noPAH high and SSc-PAH groups; there was no statistically significant trend from healthy controls to SSc-PAH $(p>0.05)$.

\section{Discussion}

Our study demonstrates that SSc patients with PAH have a gene expression profile that is distinct from those with ILD and significant restrictive disease.

When ILD patients were included, four genes plus male sex were able to predict PAH with a high degree of accuracy; 
THBS1 was excluded because it did not add sufficiently to the final model. On the other hand, after excluding patients with significant ILD, THBS1 was the most predictive gene for PAH, suggesting that, while PAH and ILD phenotypes may have overlapping pathogenic processes, there are important gene expression differences. Cheadle et al. ${ }^{7}$ compared microarray gene expression profiles in IPAH and SScPAH patients and included a small group of SSc-ILD-PH patients $(n=8)$ who exhibited a slightly different gene expression profile compared to the SSc-PAH group. However, the individual gene expression differences between those with and without ILD were not reported.

TGF- $\beta$ is central to the pathogenesis of SSc and THBS 1 is a secreted soluble peptide that is important for activation of TGF- $\beta$ signaling. ${ }^{8}$ In cell and animal models, hypoxia induces THBS1 expression in pulmonary artery smooth muscle cells, endothelial cells, and pulmonary fibroblasts in a hypoxia-inducible factor-2 $\alpha$ (HIF-2 $\alpha$ )dependent manner; these changes are associated with destabilization of endothelial cell-cell interactions, suggesting that THBS1 regulates vascular remodeling. ${ }^{9}$ Furthermore, THBS1 expression was higher in tissue from patients with end-stage PAH compared to controls 9 and mutations in THBS1 have been reported in familial PAH patients. ${ }^{10}$ Taken together, these data suggest that THBS1 is important in pulmonary vascular homeostasis and remodeling; thus, it is plausible that THBS1 is important for the development of the SSc-PAH phenotype without restrictive ILD.

S100P is a part of the S100 family of calcium-binding proteins and was strongly positively correlated to PAH. S100P is differentially expressed in PBMCs from IPAH patients ${ }^{11}$ and dimerizes with another member of the protein family, S100A1;12 S100A dimerization appears important for its function in orchestrating cardiomyocyte and endothelial function, as well as cardiac fibroblasts. ${ }^{13}$ In cardiac endothelial cells, S100A1 is involved in nitric oxide homeostasis and decreased expression is associated with arterial and $\mathrm{PH} .{ }^{13}$ Another S100A protein, S100A4, activates advance glycation end-products receptor (RAGE) leading to activation of STAT3 and decreased bone morphogenetic protein receptor-2 (BMPR2) activity in human pulmonary artery smooth muscle cells. ${ }^{14}$ In a recent multiorgan multinetwork analysis of tissues from SSc patients, S100A genes and multiple genes involved in cell cycle control, apoptosis, extracellular matrix remodeling (including TIMP1), innate immunity, and response to TGF- $\beta$ were associated with SSc-PAH and SSc-ILD. ${ }^{15}$ Therefore, we hypothesize that S100P represents a common pathogenic link between SSc-PAH and SSc-ILD, which may be mediated through interactions with S100A proteins.

CD8B1, a part of the CD8 receptor complex on cytotoxic $\mathrm{T}$ lymphocytes recognizes antigens presented by antigen-presenting cells; CD8B1 expression was negatively correlated to $\mathrm{PAH}$, suggesting decreased activity in this $\mathrm{T}$ cell population. This change in adaptive immunity may allow for the long-term remodeling seen in both ILD and PAH; however, more investigation is needed as the role of T cell biology in PAH pathogenesis remains poorly understood. ${ }^{16}$

Multiple genes were highly correlated with PAH and many of these changes were detectable in the SSc-noPAH high group (mPAP 21-24 mmHg), suggesting gene expression changes may occur early in the disease process. S100P and CD8B1 were highly correlated with PAH and chosen as inputs for both models. Moreover, gene expression changes in the SSc-noPAH high and SSc-PAH groups were very similar. However, THBS1 gene expression in the SSc-noPAH high group did not closely reflect the SScPAH group. Therefore, it is possible that SSc-ILD and SSc-PAH may share common pathogenic mechanisms early in the disease process; later, other factors, such as THBS1 expression, may promote progression toward development of more severe PAH. Thus, we may be able to use the genes identified here for early detection of both pulmonary diseases (PAH and ILD) in SSc patients.

The strengths and limitations of this study are as follows: (1) gene expression was measured with NanoString technology, which is automated, reliable, and does not depend on DNA amplification, which can result in significant noise and greater potential for error. Furthermore, a smaller number of genes were examined $(n=69)$ than in a typical microarray experiment, which reduces the number of dimensions and, therefore, the likelihood of fitting noise within our dataset (i.e. overfitting). However, NanoString technology has not yet been validated in blood biomarker development. (2) Although this was a retrospective study with limited sample size, this is, to our knowledge, the first study to specifically address gene expression differences between SSc-PAH and SSc-ILD patients; moreover, a prior study with both groups did not provide as many samples $^{7}$ nor did it report gene expression differences between these groups. (3) ILD subtypes were not defined (e.g. nonspecific interstitial pneumonitis and usual interstitial pneumonia) radiographically or histologically. However, we decided that the more clinically relevant phenotype of SSc-ILD are those patients with significant restriction and no alternative explanation, as modern high-resolution computerized tomography is sensitive enough to detect some degree of fibrosis in nearly all SSc patients. (4) RHC was not performed in all subjects without $\mathrm{PH}$, because it is difficult to justify an invasive procedure in an individual with a normal echocardiogram. The false negative rate of echocardiography for screening for $\mathrm{PH}$ is extremely low (estimated at $<1 \%$ ). In our study, $\mathrm{PH}$ was confirmed by RHC in all patients; in addition, 19 patients without $\mathrm{PH}$ underwent RHC to evaluate an abnormal echocardiogram. Thus, only 20 patients did not have an RHC and each of these individuals had a normal echocardiogram. 
There are several potential causes of elevated pulmonary pressures in SSc patients, including PAH, parenchymal lung disease, and left heart disease. In this study, we included only patients with normal pulmonary PAWP by RHC; while this does not completely exclude left heart disease, it does make it highly unlikely. Distinguishing true vasculopathy from those with PH due to ILD is challenging, which is why we examined gene expression differences between these groups. Although prospective validation is needed, our results provide a step toward a genomic profile-based distinction between these important phenotypes.

Surprisingly, our cohort had a low number of patients with anticentromere antibody. This may represent the unique characteristics of our cohort or may simply reflect the small number of patients. The frequency of anticentromere in SSc has been reported at between $20 \%$ and $30 \%$, but varies by ethnicity, the highest frequency being in northern Europeans and the lowest in non-Caucasians. In non-Caucasians, frequencies as low as $4 \%-5 \%{ }^{17}$ have been reported and while we did not record the ethnicity of our patients, our population is not predominantly Caucasian; these population differences may account for a lower prevalence of anticentromere antibodies (ACA) in our cohort. In addition, the reported frequency of antiScl-70 is $15 \%-20 \%$ in SSc in $40 \%$ of patients with diffuse cutaneous SSc and in $<10 \%$ of patients with limited SSc. In all, $4 \%-5 \%$ of our cohort had anti-Scl-70 antibodies; this is low, but again it may be due to the small number of patients and/or the predominantly limited SSc phenotype

It is not known what proportion of individuals with a borderline elevated mPAP will progress to PH. However, in this study, patients with borderline elevated mPAP exhibited a genomic profile that was not dissimilar from those diagnosed with PH. Thus, it will be interesting to follow individuals with this hemodynamic and genomic profile to determine whether they do indeed develop $\mathrm{PH}$ and whether their outcome is different than with $\mathrm{PH}$ patients or individuals without $\mathrm{PH}$.

In conclusion, SSc-PAH and SSc-ILD have similar but distinct gene expression profiles. Also, many gene expression changes occur early in the disease process, potentially allowing earlier detection. Finally, THBS1 appears to be an important mediator in the development of the PAH-predominant phenotype. Further prospective investigation is warranted.

\section{Declaration of conflicting interests}

The author(s) declared no potential conflicts of interest with respect to the research, authorship, and/or publication of this article.

\section{Funding}

This work was supported by the NIH (National Institute of Arthritis and Musculoskeletal and Skin Diseases) and Center of Research Translation (grant numbers: 1-P50-AR-060780-01 and NIAMS grant 2-R01-AR-051089-06A1).

\section{References}

1. Tyndall AJ, Bannert B, Vonk M, et al. Causes and risk factors for death in systemic sclerosis: a study from the EULAR Scleroderma Trials and Research (EUSTAR) database. Ann Rheum Dis 2010; 69(10): 1809-1815.

2. Simonneau G, Gatzoulis MA, Adatia I, et al. Updated clinical classification of pulmonary hypertension. $J$ Am Coll Cardiol 2013; 62: D34-D41.

3. Farber HW and Loscalzo J. Pulmonary arterial hypertension. N Engl J Med 2004; 351: 1655-1665.

4. Christmann RB, Hayes E, Pendergrass S, et al. Interferon and alternative activation of monocyte/macrophages in systemic-sclerosis-associated pulmonary arterial hypertension. Arthritis Rheum 2011; 63(6): 1718-1728.

5. Pendergrass SA, Hayes E, Farina G, et al. Limited systemic sclerosis patients with pulmonary arterial hypertension show biomarkers of inflammation and vascular injury. PLoS ONE 2010; 5(8): e12106.

6. Chung L, Domsic RT, Lingala B, et al. Survival and predictors of mortality in systemic sclerosis-associated pulmonary arterial hypertension: outcomes from the pulmonary hypertension assessment and recognition of outcomes in scleroderma registry. Arthritis Care Res 2014; 66(3): 489-495.

7. Cheadle C, Berger AE, Mathai SC, et al. Erythroid-specific transcriptional changes in PBMCs from pulmonary hypertension patients. PLoS ONE 2012; 7(4): e34951.

8. Lafyatis R. Transforming growth factor $\beta$ - at the centre of systemic sclerosis. Nat Rev Rheumatol 2014; 10: 706-719.

9. Labrousse-Arias D, Castillo-González R, Rogers NM, et al. HIF-2 $\alpha$-mediated induction of pulmonary thrombospondin-1 contributed to hypoxia-driven vascular remodeling and vasoconstriction. Cardiovasc Res 2016; 109: 115-130.

10. Maloney JP, Stearman RS, Bull TM, et al. Loss-of-function thrombospondin-1 mutations in familial pulmonary hypertension. Am J Physiol Lung Cell Mol Physiol 2012; 302: L541-554.

11. Ulrich S, Tarasevicience-Stewart L, Huber LC, et al. Peripheral blood B lymphocytes derived from patients with idiopathic pulmonary arterial hypertension express a different RNA pattern compared with healthy controls: a cross sectional study. Respir Res 2008; 9: 20.

12. Wang G, Zhang S, Fernig DG, et al. Heterodimeric interaction and interfaces of S100A1 and S100P. Biochem J 2004; 382: 375-383.

13. Rohde D, Busch M, Volkert A, et al. Cardiomyocytes, endothelial cells and cardiac fibroblasts: S100A1's triple action in cardiovascular pathophysiology. Future Cardiol 2015; 11(3): 309-321.

14. Meloche J, Courchesne A, Barrier M, et al. Critical role for the advanced glycation end-products receptor in pulmonary arterial hypertension etiology. J Am Heart Assoc 2013; 2: e005157.

15. Taroni JN, Greene CS, Martyanov V, et al. A novel multinetwork approach reveals tissue-specific cellular modulators of fibrosis in systemic sclerosis. Genome Med 2017; 9(1): 27.

16. Rabinovitch $\mathrm{M}$, Guignabert $\mathrm{C}$, Humbert $\mathrm{M}$, et al. Inflammation and immunity in the pathogenesis of pulmonary arterial hypertension. Circ Res 2014; 115(1): 165-175.

17. Morel PA, Chang HJ, Wilson JW, et al. HLA and ethnic associations among systemic sclerosis patients with anticentromere antibodies. Hum Immunol 1995; 42(1): 35-42. 